\title{
Über das Hirn des Chirurgen
}

Max Stäubli

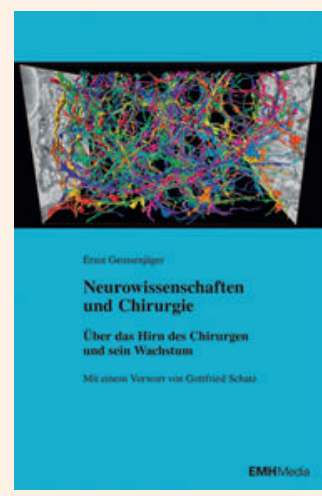

Ernst Gemsenjäger Neurowissenschaften und Chirurgie

Basel: EMH Schweizerischer Ärzteverlag; 2014. 67 Seiten, 9 Abb., davon 7 farbig, 2 Grafiken. $19.50 \mathrm{CHF}$.

ISBN 978-3-03754-079-4
Ernst Gemsenjäger arbeitet in seinem Essay die Bedingungen heraus, die der Chirurg als intelligenter Handwerker erfüllen muss, und er vergleicht sie mit andern Tätigkeiten, bei denen in den letzten Jahrzehnten eine trainingsabhängige Zunahme von Dichte und Volumen der grauen und/oder weissen Hirnsubstanz nachgewiesen wurde. Diese beruht auf einer nicht nur genetisch, sondern auch epigenetisch vermittelten Anpassungsfähigkeit der neuronalen Strukturen (Plastizität). Die Epigenetik eröffnet neue Sichtweisen und Deutungen in der anthropologischen Evolutionsbiologie, insbesondere auch der Neurowissenschaften. Gehirn-Plastizität ist die anatomische und funktionelle Adaptation an neue Anforderungen, vermittelt durch epigenetische Modulation von Genexpression. Sie wurde nachgewiesen beim Erlernen von Jonglieren, Erlernen einer Fremdsprache oder bei der Vorbereitung auf ein medizinisches Examen; Musizieren führt in mehreren Hirnregionen $\mathrm{zu}$ anatomischen und funktionellen Veränderungen. Jahrelanges Üben und Meisterschaft bilden sich im Gehirn ab. Bei Londoner Taxifahrern hat der hintere Hippocampus im Vergleich zu Kontrollpersonen ein grösseres Volumen. Diese Veränderung ist nicht Voraussetzung zur Berufswahl des Taxichauffeurs, sondern Folge des Berufs.

Nachahmung ist für das Erlernen einer Tätigkeit essentiell: Eine beobachtete Handlung kann in das eigene motorische Repertoire integriert werden dank der Aktivität der Spiegelneuronen, denen der Autor für das Erlernen der Chirurgie eine grosse Bedeutung

\section{Eine Warnung, die salamitaktischen Amputationen an der Weiterbildungszeit zugunsten von Tätigkeiten an der administrativen Front immer weiterzutreiben.}

zumisst. Spiegelneuronen werden beim Beobachter dann aktiviert, wenn er die Handlung versteht, die eine andere Person ausführt. Der Chirurg muss über ein Arsenal von sofort abrufbaren motorischen Handlungen verfügen, die ein sicheres Operieren und ein gewebeschonendes Vorgehen gestatten. Er macht die Erfahrung, dass er operative Verfahren umso leichter abschauen kann, je besser er selber schon operieren kann. Analog wurde bei Tänzern festgestellt, dass deren Spiegelneuronen umso stärker aktiviert werden, je mehr der Zuschauende mit dem Tanzprogramm schon vertraut ist.

Der Autor beruft sich auf all diese Erkenntnisse, wenn er folgert, dass es beim Chirurgen zu grundsätzlich ähnlichen anatomischen und neurophysiologi- schen Begleiterscheinungen/Funktionserweiterungen im Hirn kommt wie bei anderen Berufen, wenn das notwendige intensive Training durchgeführt wird. Gemsenjäger postuliert, dass Kompetenz und Exzellenz des Chirurgen von einem anatomischen und funktionellen Wachstum des Gehirns begleitet werden, das vermittelt wird durch Training aufgrund von erlebter Realität im Operationssaal. Nachahmen als evolutionsbiologische Ur-Erfahrung.

Die Verantwortlichen müssen dem wichtigsten Faktor, der Zeit, die der angehende Chirurg im Operationssaal zubringen muss, voll Rechnung tragen. Eine schlechte Ausbildung ist auch kostentreibend. Gute Qualität ist in der Chirurgie das höchste Gut für alle Beteiligten. Ein stures Arbeitszeitdiktat könne sich deshalb als kontraproduktiv erweisen.

Obschon direkte Untersuchungen fehlen, gibt es für mich keinen Zweifel, dass die Lernprozesse beim Chirurgen in ähnlicher Weise Erweiterungen der Hirnstruktur und -funktion bewirken bzw. erfordern wie in anderen Berufen. Wenn auch unbeantwortet, ist die Frage interessant, ob die in der operativen Tätigkeit erworbenen chirurgischen Fähigkeiten zu den vererbbaren epigenetischen Veränderungen gehören, eine Frage, die auch Gottfried Schatz im Vorwort aufwirft.

Es war höchste Zeit, dass der erfahrene ehemalige Chef-Chirurg einer Weiterbildungsstätte davor warnt, die salamitaktischen Amputationen an der Weiterbildungszeit zugunsten von Tätigkeiten an der administrativen Front immer weiterzutreiben. Diese Überlegung gilt auch für andere medizinische Fächer.

Als Internist beschlichen mich bei der Besprechung dieser Arbeit dennoch Zweifel an meiner Zuständigkeit. Weil aber auch die Innere Medizin planmässiges Vorgehen, Geschicklichkeit und ein Arsenal an unmittelbar verfügbaren Vorgehensweisen - Wissen, Können, Erfahrung - verlangt, die z. B. über Leben und Tod entscheiden, denke ich, dass das für den Chirurgen Postulierte auch für den Internisten gilt: Die Innere Medizin muss von guten Tutoren vorgelebt und über einen Zeitraum von Jahren gelernt werden. Früher sprach man von Vorbildern. Nicht nur im Szenarium «Operationssaal» ist der Faktor des sich Einfühlens in das Handeln des Lehrers bedeutsam. Nachahmen hat in den Situationen Lehrer/Schüler in der Medizin einen sehr hohen Stellenwert. Und niemand spricht hier von Plagiat.

Der Text ist in konzisem Stil ohne sprachlich störendes Beiwerk verfasst. Es handelt sich um ein schmuckes Bändchen mit repräsentativen, meist farbigen und einprägsamen Abbildungen.

Ich kann es auch jenen herzlich empfehlen, für die die Chirurgie ein Fremdfach geblieben ist, sowie den nichtmedizinischen Spitalkadern. 